\title{
Estimation of Currents with Acoustic Navigation Beacons
}

\author{
José Melo, Nuno Cruz, Rui Almeida \\ INESC TEC \\ and Faculty of Engineering, \\ University of Porto, Portugal \\ \{jose.melo, nacruz, rui.almeida\}@ @e.up.pt
}

\begin{abstract}
In this article we introduce a method to estimate the water current in AUV operation scenarios. The method is based on reciprocal sound transmission between pairs of acoustic navigation beacons, with a minimum of three beacons being required to obtain an estimate of both current velocity and direction. We analyse the sensitivity of the current estimation with respect to the geometry of the beacon location. We demonstrate the applicability of the method with a case study.
\end{abstract}

\section{INTRODUCTION}

Acoustic Navigation is still the main absolute non-drifting navigation technique for Unmanned Underwater Vehicles (UUVs). Acoustic Navigation embraces a number of techniques that rely on the exchange of acoustic signals between a vehicle, which the position needs to be derived, and a set of existing acoustic beacons. Broadly speaking, three distinct Acoustic Navigation schemes exist, namely the Long Baseline (LBL), the Short Baseline (SBL), and the Ultra Short Baseline (USBL). The major difference between these schemes is the length of the baseline, which is the distance between the acoustic beacons. Comparing to their counterparts, one of thee main advantages of LBL systems is that they grant navigation capabilities in a wide area and have very good, depth independent, position accuracy, which falls in the meter scale. Because of that, LBL positioning systems have always played a major role in the field of underwater robotics. In this article, we will be focused exclusively on those systems.

On LBL navigation, the beacons are deployed on predetermined fixed positions, whether underwater or at the surface. It is possible to derive ranges between vehicle and the beacons from the Time-Of-Flight (ToF) of the acoustic signals. These ranges can then be used with multilateration techniques to obtain the position of the vehicle. However, this process is dependent on knowing the speed of sound in the area of operations. While roughly speaking the speed of sound is around $1500 \mathrm{~ms}^{-1}$, this value is highly dependent of environmental conditions, like temperature and salinity of the water, but also water currents that may exist. Therefore, being able to estimate the local speed of sound, but also the current speed and direction can be of extreme importance to obtain high precision navigation and tracking solutions for UUVs.

In this article we introduce a method that makes use of the acoustic signals exchanged by a set of three acoustic navigation beacons to estimate the speed of sound and water currents that might exist. Our method is based on reciprocal travel time of acoustic signals between pairs of acoustic beacons. While similar methods have been described in the literature, the focus of this work is on extending the features of traditional acoustic beacons, using them to also estimate horizontal currents.

This article is organized as follows. In the next section estimation of currents with reciprocal travel time will be introduced. After that, in Section III we will present the standard LBL configuration of acoustic navigation beacons, and formulate the problem of current estimation. In Section IV a sensitivity analysis of the problem will be formulated, and Section V presents a case study for applying the proposed method. Finally, Section VI will draw some conclusions of what has been presented.

\section{ReCiProcAl TRAVEL Time FOR CURRENT ESTIMATION}

The estimation of precise current profiles can be done by inversion of tomographic data. However, this requires solving challenging inverse problems, which involve high computation power. An alternative to such approaches is to use reciprocal sound transmissions to obtain range-averaged estimates for the current. This has been previously demonstrated in the literature, initially in [1] and later confirmed for example in [2]. Reciprocal sound transmissions is a method that compares the travel time difference of an acoustic signal between two fixed points when travelling in opposite directions. By knowing the distance between the two points, it is possible to estimate the averaged speed of sound, and any currents that might affect it.

Considering that $t_{A B}$ is the ToF of an acoustic signal from beacon $A$ to a beacon $B$, one can write:

$$
t_{A B}=\frac{d}{v_{s}+v_{c}}
$$

where $d$ refers to the distance between the two points, $v_{s}$ to a local homogeneous speed of sound and $v_{c}$ to the current. Analogously, and considering that in the opposite direction $v_{c}$ contributes negatively to the ToF, one can write:

$$
t_{B A}=\frac{d}{v_{s}-v_{c}}
$$


By algebraic manipulation of (1) and (2), it is possible to solve for the speed of sound and currents:

$$
\begin{aligned}
& v_{s}=\frac{d}{2}\left(\frac{1}{t_{A B}}+\frac{1}{t_{B A}}\right) \\
& v_{c}=\frac{d}{2}\left(\frac{1}{t_{A B}}-\frac{1}{t_{B A}}\right)
\end{aligned}
$$

For the case when no currents are present, $t_{A B}=t_{B A}$, that is the time travel time measured from each of the beacons are equal, and therefore $v_{c}=0$. However, in the general case, such condition does not hold.

While the above equations are a simple way to obtain values for both speed of sound and currents with the reciprocal sound transmission method, they are only valid if $A$ and $B$ are aligned with the direction of the currents. In alternative, the expected direction of the currents can also be taken in consideration. This is the case in the majority of the examples in literature, as for example in [3] or [4], where the reciprocal travel time method is used to predict the currents of rivers, where its direction is easy to predict. On the general case, however, this is not true and the reciprocal method can only be used to establish $v_{c_{A B}}$, which is the projection of the current $v_{c}$ in the $A B$ direction.

When the current has an unknown direction, the reciprocal travel time method for sound transmission can only be used if the travel time between three different points are available. The reason for this is related with the three unknowns that need to estimated, namely sound velocity $v_{s}$, and the two components of the current velocity, $v_{c_{N}}$ and $v_{c_{E}}$, respectively the projections of the current vector on both the North and East coordinated axis. In the next section we will detail the implementation of the proposed algorithm using acoustic navigation beacons.

\section{Acoustic Navigation Beacons}

The proposed algorithm for the experimental determination of currents requires the use of acoustic navigation beacons. While traditionally the beacons that compose an LBL network were deployed on the sea bottom, the use of GPS enabled buoys that are deployed at the sea surface is gaining popularity, mostly due to it's ease of calibration, deployment and recovery of the equipment. Figure 1 depicts such kind of beacons, which will be the focus of our approach. Common configurations in this kind of beacons include a GPS receiver, WiFi or radio connections for communications, and an acoustic communication transceiver, which is able to timely transmit and receive acoustic signals. The interested reader should refer to [5] for a detailed description.

The knowledge of existing currents can be decisive for accurately determining the position of AUVs. Nevertheless, the existence of currents is often overlooked when characterizing the precision of acoustic navigation systems, as for example in [6]. At the same time, such knowledge can be important for planning and accurate control of UUVs. In what follows we will derive a method to determine such currents, should they exist, using acoustic navigation beacons.

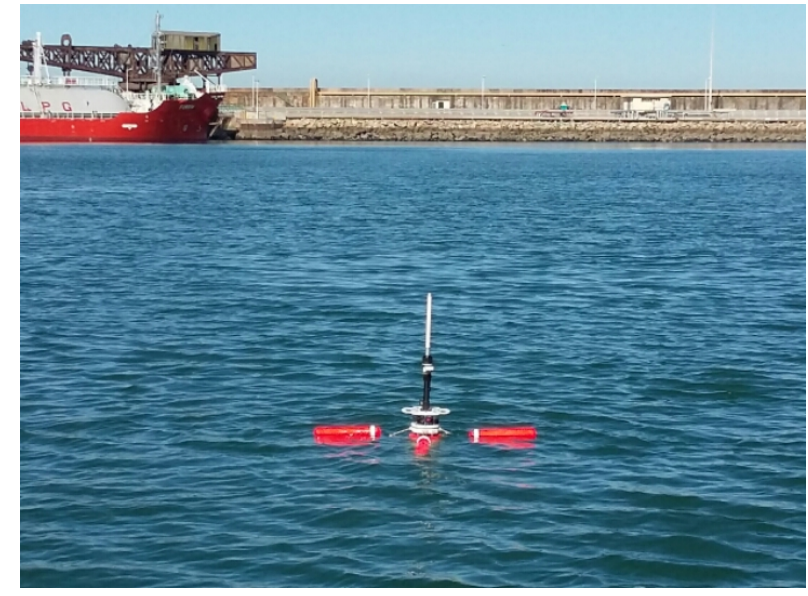

Fig. 1. Example of an acoustic navigation beacon, foreseen to be used with the proposed method.

The proposed method for determining the $2 \mathrm{D}$ currents is dependent on both the position of all the beacons, and also the travel time of the acoustic signals exchanged in the network. Due to the geometric constraints of the problem, at least three beacons deployed in non-collinear directions are needed in order to determine the magnitude of the water currents, but also its direction.

We consider the case where the acoustic navigation beacons $A$ and $B$ have already been deployed, constituting the baseline $\overline{A B}$, with a given angle $\gamma$ with the North direction. As a rule of thumb, when operating with three navigation beacons, it is usually advantageous to have the third beacon located in the perpendicular bisector of the baseline $\overline{A B}$. With such configuration, $\angle C A B=\angle C B A=\alpha$. Figure 2 presents a schematic diagram of the setup we propose to estimate horizontal currents.



Fig. 2. Geometric diagram for the experimental setup

\section{A. Current Estimation}

The current $v_{c}$ that we want to estimate can be decomposed on its components according to the North and East coordinates, $v_{c n}$ and $v_{c e}$ respectively. These components of the current can 
be expressed as a combination of the projection of $v_{c}$ on the different directions between each pair of beacons, $v_{c_{A B}}, v_{c_{B C}}$ and $v_{c_{A C}}$ respectively. This can be done by taking into account the angle $\gamma$, which corresponds to the angle of the baseline $\overline{A B}$ relative to the North direction, and the angle $\alpha$, between the baseline and the directions going from each of the beacons $A$ and $B$, and the third beacon $C$. This is illustrated in Figure 2. Such geometrical relationships can be expressed in matrix form:

$$
V_{m}=\Theta V_{c}
$$

$\boldsymbol{V}_{\boldsymbol{m}}=\left[\begin{array}{lll}v_{c_{A B}} & v_{c_{B C}} & v_{c_{A C}}\end{array}\right]^{T}$ is the matrix of the measured projected currents, and $\boldsymbol{V}_{\boldsymbol{c}}=\left[\begin{array}{ll}v_{c n} & v_{c e}\end{array}\right]^{T}$ is the matrix of the current components we wish to determine. Naturally, $\Theta$ is the matrix of the geometrical projections between the beacons directions and the current. By noting that, due to symmetry $\left|v_{c_{A C}}\right|=\left|v_{c_{B C}}\right|=v_{c \alpha}, \Theta$ can be expressed in terms of the angles $\alpha$ and $\gamma$ as

$$
\Theta=\left[\begin{array}{cc}
\cos (\gamma) & \sin (\gamma) \\
\cos (\pi-(\gamma+\alpha) & \sin (\pi-(\gamma+\alpha) \\
\cos (\gamma-\alpha) & \cos \left(\frac{\pi}{2}-(\gamma-\alpha)\right)
\end{array}\right]
$$

By using a Least Square Estimator the components of the current, and therefore it's amplitude and direction, can be obtained, using for example the normal equations of Least Squares, so that

$$
V_{c}=\left(\Theta^{T} \Theta\right)^{-1} \Theta^{T} V_{m}
$$

$\Theta$ determines the geometry of the problem, but also plays a significant role on the perturbation analysis of the least squares solution. In fact it can be demonstrated that $\Theta$ determines the sensitivity to the measurements $V_{m}$. Therefore it is important to ensure that the condition number of $\Theta, \kappa(\Theta)$, always remains low, ensuring that small perturbations of $V_{m}$ will not cause big perturbations in $V_{c}$. When this happens, $\Theta$ is said to be a well-conditioned matrix. This is of particular importance given the possible values for $\alpha$ and $\gamma$.

\section{Sensitivity Analysis}

In the previous sections it was already detailed how the reciprocal travel time method can be used to estimate horizontal currents using acoustic navigation beacons. As mentioned, we are focused on studying the position of a third beacon relative to its baseline, and how it affects the measurement process. In this section, a sensitivity analysis of such problem will be performed, focused on minimizing the influence of different parameters on $v_{c \alpha}$, as the position of the third beacon has no influence in $v_{c_{A B}}$. In specific, we will be studying the influence of the distance between each two beacons, but also on the angle $\alpha$ between the baseline and the third beacon, and its position uncertainty.

\section{A. Distance}

Recalling (4), the expression for measured currents, it can be refined by noting that $t_{A B}=t$ and $t_{B A}=t+\Delta t$. That is, the travel time measured from each of the beacons differs by an amount of $\Delta t$ seconds, which is a caused by existing water currents. Substituting in (4), and considering $t>>t$, we have:

$$
v_{c}=\frac{d}{2}\left(\frac{t_{A B}-t_{B A}}{t_{A B} t_{B A}}\right) \approx \frac{d \cdot \Delta t}{2 t^{2}}
$$

By further considering the time $t$ as a function of both the distance $d$ and nominal speed of sound $v_{s}$, we arrive to

$$
v_{c}=\frac{-\Delta t \cdot v_{s}^{2}}{2} \cdot \frac{1}{d}
$$

which expresses the inversely proportional dependency of the water currents determination with the distance between each of the beacons. This relationship is also dependent on the squared value of the speed of sound.

Starting from (9), and by shrinking $\Delta t$ to $\tau$, the minimum time quantity that can be measured, we arrive to the minimum current that can be measured. Such value, the sensitivity of the system, is basically limited by $\tau$, the resolution with which is possible to measure $t$. As expected, the precision in measuring time is of uttermost importance to the estimation of the currents.

Because we are measuring absolute differences of travel times, increasing the distance between the beacons will have as a consequence an increase in the sensitivity of the measurements, which will have an impact on the smallest possible current that can be measured. That means when the distance between any two beacons is longer, it is possible to measure smaller variations in the respective current projection.

\section{B. Angle}

As mentioned before, when using acoustic navigation beacons to determine the currents, what is sought is where to position the beacon $C$ in the perpendicular bisector of the baseline $\overline{A B}$, guaranteeing that it favours the determination of the currents. Analysing Figure 2, it is clear that the position of beacon $C$ can be expressed in terms of the angle $\alpha$ between the baseline beacons and a third beacon.

We start by analysing the effect of $\alpha$ on the sensitivity of the measurements. Recalling (9), distance $d$ can be written as a function of the baseline $l$ and the angle $\alpha$ :

$$
v_{c \alpha}=\frac{2 v_{s}^{2} \tau}{l} \cos (\alpha)
$$

This expression for $v_{c}$ is basically a cosine function, affected by a constant that is dependent on both the speed of sound $v_{s}$, and $\tau$. From (10) one can note that it would be beneficial to position the third acoustic beacon in a way that the angle $\alpha$, between the baseline and this beacon would be high, as this would increase the sensitivity of the system.

\section{Position}

It is also important to consider the influence that the position of the beacons, and its variations might have on measuring water currents. This is particularly important for the case when using beacons at the surface, and with positions given by Global Navigation Satellite Systems (GNSS), such as GPS, 
Galileo, etc.. In fact, positions given by GNSS receivers always have some associated uncertainty, which should naturally be reflected on the estimation of the water currents.

First, the sensitivity of the measurement equation is derived with respect to variations of the position, $p$. By differentiating (10) with respect to the position, we obtain

$$
\frac{\partial v_{c \alpha}}{\partial p}=\frac{-2 v_{s}^{2} \tau}{l} \sin (\alpha) \cdot \delta_{\alpha}
$$

where $\delta_{\alpha}$ refers to the variations of $\alpha$ induced by uncertainty in the GNSS obtained position.

There are different statistical measures to account to such uncertainties in the horizontal position associated with GNSS systems, like the Distance Root Mean Squared (DRMS), the Circular Error Probability (CEP). Both of these metrics refer to the radius of a circle, centred at the true position, and containing a given percentage of the obtained estimated positions. For example $C E P_{50}, C E P_{95}$ and $C E P_{99}$ refer to a radius containing, respectively, $50 \%, 95 \%$ and $99 \%$ of the estimated positions.

We adhere to such notion, by considering that the GNSS obtained position of the beacons is associated with a given uncertainty, so that the true position of the beacons is within a circle, with a radius given by $\sigma_{G N S S}$. By bounding the position of the beacons to this circle, it is possible to reflect uncertainty in the positions to a worst case scenario uncertainty in $\alpha$. Considering the geometric properties of the problem, it is then possible to write $\delta_{\alpha}$ as:

$$
\delta_{\alpha}=\arcsin \left(\frac{2 \sigma_{G N S S}}{l \cos (\alpha)}\right)
$$

\section{CAse Study}

In this section we will perform a case study analysis of a given acoustic navigation system, illustrating how the proposed method can be used to measure water currents.

We start by studying the effect of varying the distance between beacons. Figure 3 shows this relation between sensitivity and distance. This relation is plotted for different values of $\tau$, which represent the timing precision of the acoustic system. The plot clearly demonstrates that the increase of distance between pairs of beacons can increase the sensitivity of the measurements, but is the $\tau$ that plays a determinant role on it, therefore being desirable to have $\tau$ as low as possible.

In the remainder of this case study, the focus will be on planning the the acoustic beacons distribution for a system that is able to achieve sensitivities of up to $0.1 \mathrm{~m} / \mathrm{s}$, and characterized by having $\tau=10 \mu \mathrm{s}$ and a baseline length $\overline{A B}$ set to be $300 \mathrm{~m}$. The different parameters of possible LBL configuration with 3 acoustic navigation beacons, as mentioned in the previous sections, will be defined accordingly.

It is important to understand whether $\Theta$ is a wellconditioned matrix. If this would be the case, small variations in the inputs, in this case the measured project currents, will only cause small variations in the output, the components of $v_{c}$, and. For that we study the condition number associated to both $\Theta$ and $\left(\Theta^{T} \Theta\right)$, associated with its Moore-Penrose

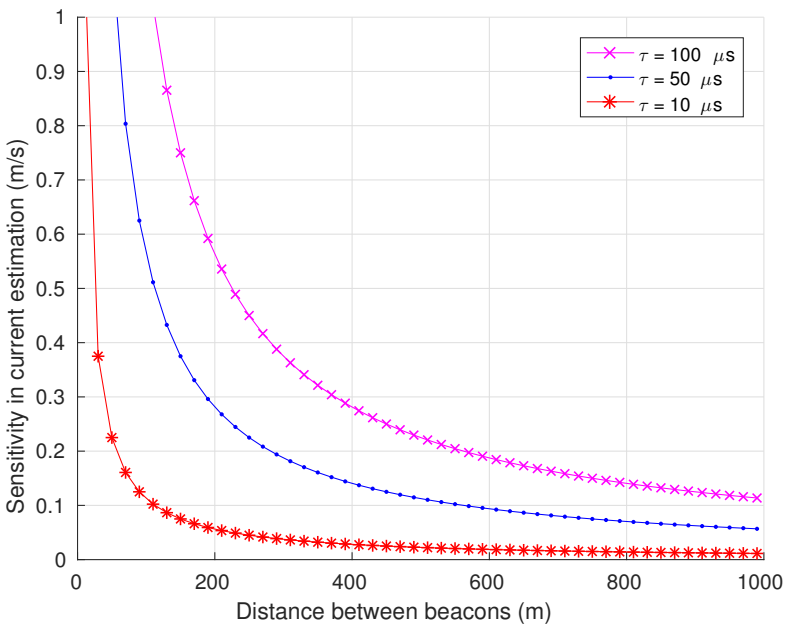

Fig. 3. Sensitivity of current measurements with the distance between the beacons, for different values of $\tau$.

pseudo-inverse and relevant for the Least Squares Estimator (LSE). Recalling from Section III, the angle $\alpha$, refers to the angle between the baseline and the third beacon, while $\gamma$ refers to the orientation of the baseline with respect to the true North. Figure 4 illustrate the variation of both $\kappa(\Theta)$ and $\kappa\left(\Theta^{T} \Theta\right)$, for an example situation where $\gamma=90^{\circ}$.



Fig. 4. Condition number of the matrix $\Theta$ associated with the geometric projections of the measured currents.

As can be seen in Figure 4, as the angle $\alpha$, between the baseline and this beacon, decreases, the conditioning of the matrix $\Theta$ gets worse. The same behaviour happens with $\Theta^{T} \Theta$, but in this case more dramatically. Therefore, in order to favour the stability of the LSE, it is important to keep $\alpha>30^{\circ}$.

We then study the effect of angle $\alpha$. Naturally, with the baseline length fixed and the beacon $C$ situated over the perpendicular bisector of the baseline, the value of $\alpha$ will influence the distance between the beacons $A$ and $B$ of the baseline, and the third beacon $C$. Figure 5 display the effect that $\alpha$ has in the sensitivity of the system. As $\alpha$ approaches 
$90^{\circ}$, the distance between beacons will increase, therefore decreasing the sensitivity. As can be seen in the Figure, for an achievable sensitivity of at least $0.1 \mathrm{~m} / \mathrm{s}, \alpha$ must be higher than about $45^{\circ}$. Naturally, the higher the angle, the better the sensitivity of the system.

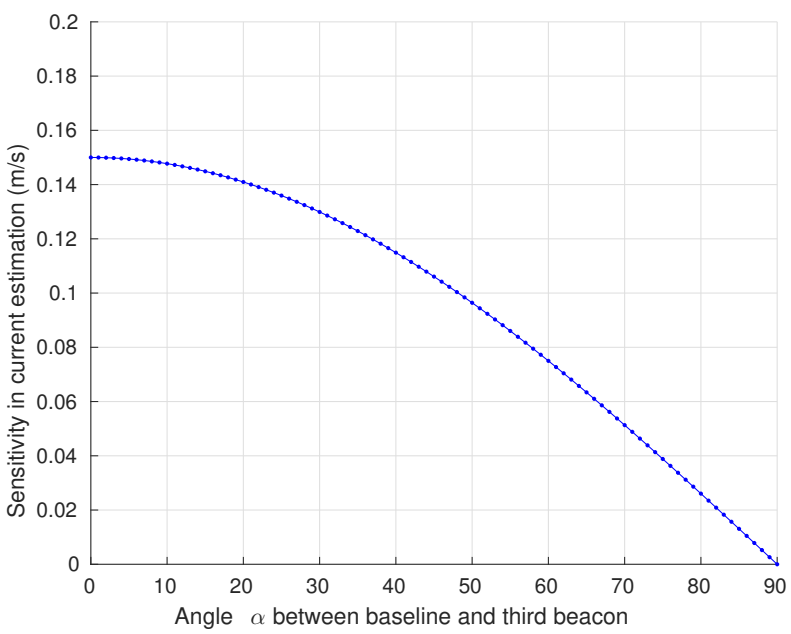

Fig. 5. Variation of the sensitivity of the system with respect to the angle $\alpha$.

We continue the analysis by studying how the uncertainty in the position can be reflected in an uncertainty in $\alpha$. For that, it is assumed a $\sigma_{G N S S}=1 \mathrm{~m}$. This is a value in line with what some advanced GNSS receivers can achieve in single mode positioning, and well above what can be achieved for differential or real-time kinematic modes with high-end receivers. Figure 6 shows how this level of uncertainty can affect uncertainty in $\alpha$.



Fig. 6. Uncertainty in the angle $\alpha$ cause by uncertainty in the position of the beacon.

Consistent to the geometric constraints of the problem, for higher angles $\alpha$ the same uncertainty in position will cause higher perturbations $\Delta_{\alpha}$. At the same time, the higher the $\Delta_{\alpha}$, the higher the induced variations in $v_{c \alpha}$. This is naturally an undesirable scenario, as only the uncertainty in the GNSS derived positions will induce variations in the estimated currents, as illustrated in Figure 7. Therefore, we should choose $\alpha$ so that $\Delta v_{c \alpha}$ are minimum.



Fig. 7. Uncertainty in $v_{c \alpha}$ caused by uncertainty in $\Delta_{\alpha}$.

Taking in consideration the analysis above, it is clear that the angle $\alpha$, and therefore the position of beacon $C$, will have a significant impact on the accuracy of the estimated currents. Increasing $\alpha$ will improve the sensitivity of the system, as the distance between beacons also increase. On the other hand, the further apart the beacons are, the higher the influence of the position uncertainty in $\alpha$, which in turn will have a dramatic effect in $\Delta v_{c}$, as illustrated in Figure 7.

In order to make a decision about the angle $\alpha$, between the baseline and the third beacon, the expected achievable sensitivities assumed for this case study, of $0.1 \mathrm{~m} / \mathrm{s}$, need to be taken in consideration. As such, going back to Figures 5 and 7 , the angle $\alpha$ should be chosen so that the sensitivity to the different parameters remains below the expected limit. From Figure 5, it can be concluded that $\alpha>50^{\circ}$. Analogously, from Figure 7, it can be concluded that $\alpha<60^{\circ}$. Therefore, one can conclude that for best performance, beacon $\mathrm{C}$ should be in a position along the baseline bisector so that the angle $50^{\circ}<\alpha<60^{\circ}$. Incidentally, this also corresponds to a situation where $\kappa(\Theta)$ is close to its minimum.

\section{CONCLUSION}

This article introduced a method that takes advantage of acoustic navigation beacons used in AUV operations to estimate water currents. Typically, such operations require a minimum of two beacons for AUV navigation. Our method is based on reciprocal sound transmission between pairs of acoustic navigation beacons, with a minimum of three beacons being required to obtain an estimate of both current velocity and direction. We formulate our problem as finding the location of a third beacon, that complements a two-beacon baseline and optimizes sensitivity of the estimated current.

Such optimization requires accurate knowledge of beacon location and precise timing of the exchanged acoustic signals. 
A sensitivity analysis of these parameters has been performed, and their effects on the obtained solution has been studied. We propose a practical method of combining both sensitivities to compute an area for the deployment of the third beacon, ensuring that the overall uncertainty falls within predefined levels.

As far as future work is concerned, the developed method will be experimentally validated in real-world scenarios, following a similar approach that was used in previous work by the same authors [6]. Prior work has demonstrated that knowledge of the currents plays a major role in the accuracy of AUV navigation systems. Therefore, we will integrate the developed procedure with different planning and navigation strategies, to improve overall performance of AUV missions.

\section{ACKNOWLEDGEMENTS}

This work is financed by the ERDF - European Regional Development Fund through the Operational Programme for Competitiveness and Internationalisation - COMPETE 2020 Programme within project «POCI-01-0145-FEDER-006961», and by National Funds through the Portuguese funding agency, FCT - Fundação para a Ciência e a Tecnologia as part of project «UID/EEA/50014/2013».

\section{REFERENCES}

[1] H. Zheng, N. Gohda, H. N. H, T. Ito, H. Yamaoka, T. Tamura, Y. Takasugi, and A. Kaneko, "Reciprocal sound transmission experiment for current measurement in the Seto Inland Sea, Japan," Journal of Oceanography, vol. 53, pp. 117-128, April 1997.

[2] Y. Adityawarman, A. Kaneko, K. Nakano, N. Taniguchi, K. Komai, $\mathrm{X}$. Guo, and N. Gohda, "Reciprocal sound transmission measurement of mean current and temperature variations in the central part Aki-nada of the Seto Inland Sea, Japan," Journal of Oceanography, vol. 67, no. 2, pp. 173-182, 2011.

[3] C. Zhang, X. H. Zhu, A. Kaneko, Q. Wu, X. Fan, B. Li, G. Liao, and T. Zhang, "Reciprocal sound transmission experiments for current measurement in a tidal river," in Proc. of MTS/IEEE Oceans'10 Conference, Sidney, Australia, May 2010, pp. 1-5.

[4] Y. H. Chen, N. Taniguchi, C. T. Liu, and C. F. Huang, "Acoustic current measurement using travel-time method in Bachimen Harbor, Taiwan," in Proc. of the MTS/IEEE OCEANS'14 Conference, Taipei, Taiwan, April 2014, pp. 1-5.

[5] R. Almeida, N. Cruz, and A. Matos, "Man portable acoustic navigation buoys," in Proc. of MTS/IEEE Oceans'16 Conference, Shanghai, China, April 2016, pp. 1-6.

[6] R. Almeida, J. Melo, and N. Cruz, "Characterization of measurement errors in a LBL positioning system," in Proc. of MTS/IEEE Oceans'16 Conference, Shanghai, China, April 2016, pp. 1-6. 